\title{
CALCULATING THE RESPONSE OF AN OSCILLATOR TO ARBITRARY GROUND MOTION*
}

By George W. Housner

IN COMPUTING the response of a simple oscillator to arbitrary ground motion it is necessary to use arithmetical or graphical methods of solution. ${ }^{1}$ A direct arithmetical or graphical solution of the differential equation of motion will give the response of the oscillator, but the method given by Lord Rayleigh ${ }^{2}$ affords a considerable saving in labor.

This method uses the "principle of superposition," which states that if two exactly similar oscillators are each acted upon by a different force, adding the

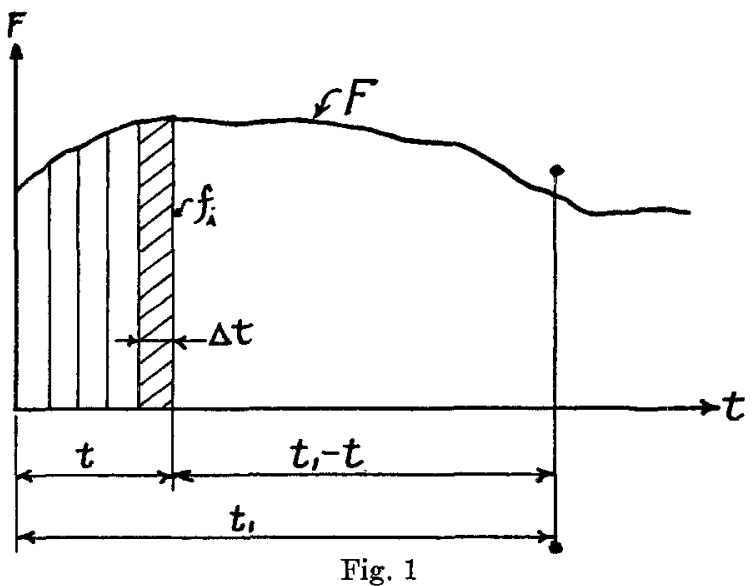

motion of the first ascillator to that of the second oscillator results in the same motion that would occur if both forces acted upon a single oscillator. Designating the motion caused by force $F_{1}$ as $M_{\left(F_{1}\right)}$ and that caused by $F_{2}$ as $M_{\left(F_{2}\right)}$, the principle of superposition states that

$$
M_{\left(F_{1}\right)}+M_{\left(F_{2}\right)}=M_{\left(F_{1}+F_{2}\right)}
$$

By induction this result can be extended to any number of forces

$$
M_{F_{1}}+M_{F_{2}}+M_{F_{3}}+M_{F_{4}}+\ldots+M_{F_{N}}=M_{F_{1}+F_{2}+F_{3}+F_{4}} \ldots+M_{F_{N}}
$$

The case of an oscillator subjected to arbitrary ground motion is the same as that of an oscillator acted upon by a continuously varying force $F$ (the ground acceleration). So if the force $F$ is subdivided into the increments $f_{i}$ (fig. 1),

* Manuscript received for publication May 2, 1940.

1 Methods of computing the responses of oscillators are described in this paper. Mechanical methods have been used by a number of investigators, e.g., U. S. Bureau of Reclamation, Denver; L. S. Jacobsen, Stanford University; M. A. Biot, Columbia University; A. Ruge, Mass. Inst. of Tech.

2 Theory of Sound, Vol. 1, p. 74. 
the response of an oscillator to $F$ is equal to the sum of the responses of a number of oscillators each of which is acted upon by one of the elemental impulses $f_{\text {. }}$

$$
M_{F}=M_{f_{1}}+M_{f_{2}}+\ldots+M_{f_{n}}
$$

If $F$ is so subdivided that each force $f_{i}$ acts only for a short interval $\Delta t$, the effect is to give each oscillator an initial velocity, after which it vibrates with simple harmonic motion. The equation of simple harmonic motion for an oscillator starting with zero displacement is $y=\frac{v_{0}}{p} \sin p t$, where $y=$ displacement, $v_{0}=$ initial velocity, $t=$ time, and $p=\sqrt{\frac{k}{m}}$, where $k=$ spring constant and $m=$ mass. The velocity caused by the force $f_{i}$ acting for a time $\Delta t$ is given by the equation of impulse and momentum

$$
f_{\imath} \Delta t=m v
$$

or $v=\frac{f_{i}}{m} \Delta t$ and the displacement at time $t_{1}$ due to the force $f_{i}$ acting at time $t_{i}$ (fig. 1) is

$$
y_{\imath}=\frac{f_{i}}{m} \frac{\Delta t}{p} \sin p\left(t_{1}-t_{i}\right)
$$

Adding the displacements caused by each of the $f_{i}$ 's, the displacement at time $t_{1}$ of an oscillator acted upon by $F$ is given by

or, passing to the limit

$$
y=\sum_{i} \frac{f_{i}}{m p} \sin p\left(t_{1}-t_{i}\right) \Delta t
$$

$$
y=\int_{0}^{t_{1}} \frac{F}{m p} \sin p\left(t_{1}-t\right) d t
$$

The magnitude of the force $F$ is given by $F=m \ddot{x}$, where $\ddot{x}=\frac{d^{2} x}{d t^{2}}=$ the ground acceleration and $x=$ ground displacement. Substituting this value for $F$ gives

$$
y=\int_{0}^{t_{1}} \frac{\ddot{x}}{p} \sin p\left(t_{1}-t\right) d t
$$

Integrating this by parts twice gives

$$
\begin{aligned}
y & =-\frac{\dot{x}_{0}}{p} \sin p t_{1}+\int_{0}^{t_{1}} \dot{x} \cos p\left(t_{1}-t\right) d t \\
& =-\frac{\dot{x}_{0}}{p} \sin p t_{1}-x_{0} \cos p t_{1}+x-p \int_{0}^{t_{1}} x \sin p\left(t_{1}-t\right) d t
\end{aligned}
$$


If at time $t=0$ the initial ground displacement $x_{0}=0$ and the initial ground velocity $\dot{x}_{0}=0$, this equation reduces to

$$
y=x-p \int_{0}^{t_{1}} x \sin p\left(t_{1}-t\right) d t
$$

Either equation 1 or equation 3 may be used to determine the response of an oscillator. Equation 1 uses the ground acceleration $(\ddot{x})$ and equation 3 uses the ground displacement $(x)$. In general, equation 3 is preferable, since the ground displacement is more regular than the ground acceleration, which simplifies the numerical computation. However, since the available strong-motion records have been recorded by accelerographs the record must be integrated twice to give ground displacements. Also, owing to the time lag in starting, the record often begins after the start of the ground motion, so that at time $t=0$ on the record the acceleration, velocity, and displacement are not zero. This, then, requires the use of equation 2 instead of equation 3 , so that the simplification due to greater regularity of the ground displacement is offset by the necessity for double integration of the accelerograph record and the use of equation 2 . Responses of oscillators have been calculated, using both ground acceleration and ground displacement. The use of the ground acceleration has proved to be the more satisfactory.

Using equation 1

$$
y=\frac{1}{p} \int_{0}^{t_{1}} \ddot{x} \sin p\left(t_{1}-t\right) d \dot{t}
$$

to determine the response of an oscillator; since $\ddot{x}$ and $p$ are known quantities, the integrand may be computed for given $t_{1}$ and the integration carried out either arithmetically or graphically. This gives the oscillator displacement for a specific time $t_{1}$, so the integration must be repeated for other $t_{1}$ 's until a sufficient number of points has been computed to allow the response curve to be drawn.

This procedure can be simplified by rewriting equation 1 by trigonometric formula.

or

$$
y=\frac{1}{p} \int_{0}^{t_{1}} \ddot{x}\left(\sin p t_{1} \cos p t-\cos p t_{1} \sin p t\right) d t
$$

$$
y=\frac{1}{p} \sin p t_{1} \int_{0}^{t_{1}} \ddot{x} \cos p t d t-\frac{1}{p} \cos p t_{1} \int_{0}^{t_{1}} \sin p t d t
$$

When $t_{1}=(n+1 / 2)(n=$ an integer $), \cos p t_{1}=0$ and $\sin p t_{1}= \pm 1$, so for these times equation 4 reduces to

$$
y=\frac{1}{p} \int_{0}^{t_{1}} \ddot{x} \cos p t d t
$$


Similarly, when $t_{1}=n \pi$ equation 4 reduces to

$$
y=-\frac{1}{p} \int_{0}^{t_{1}} \ddot{x} \sin p t d t
$$

Plotting $\frac{\ddot{x}}{p} \cos p t$ and $\frac{\ddot{x}}{p} \sin p t$ to a convenient scale and integrating each of these, the oscillator displacement at times $t_{1}=(n+1 / 2) \pi$ and $n \pi$ are given directly, and for times between these it is only necessary to multiply the ordinates of the curves at these points by sine $p t_{1}$ and cosine $p t_{1}$, respectively, and add. Thus two integrations will completely determine the response of the oscillator.

A variation of this method is derived by noting that equation 4 may be

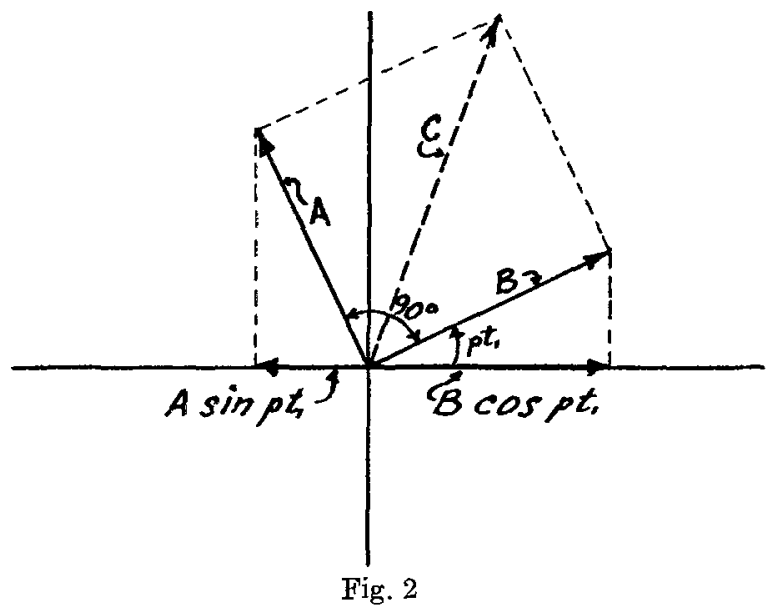

represented as the sum of the projections upon an axis of two rotating vectors $90^{\circ}$ out of phase (fig. 2)

$$
\begin{aligned}
& \text { Vector } A=\frac{1}{p} \int_{0}^{t_{1}} \ddot{x} \cos p t d t \\
& \text { Vector } B=-\frac{1}{p} \int_{0}^{t_{1}} \ddot{x} \sin p t d t
\end{aligned}
$$

$A \sin p t_{1}+B \cos p t_{1}$ is equal to the projection on the axis of $C$ where $C$ is the vector sum of $A$ and $B\left(C=\sqrt{A^{2}+B^{2}}\right)$. As $t_{1}$ increases, the angle $p t_{1}$ increases and the vectors rotate about the origin. When $C$ is coincident with the axis, $A$ $\sin p t_{1}+B \cos p t_{1}=C$, that is,

$$
y=\sqrt{A^{2}+B^{2}}
$$

$C$ coincides with the axis twice in each interval $n \pi<p t_{1}<(n+2) \pi$, the exact time of coincidence being dependent upon the location of the point from which the time is measured $(t=0)$. 
The displacement $y$ is always less than or equal to $C$, so plotting the values of $\pm C$ will give an envelope of the maximum oscillator displacement (fig. 3).

Since

$$
C=\sqrt{A^{2}+B^{2}}=\left(\left(\frac{1}{p} \int_{0}^{t_{1}} \ddot{x} \cos p t d t\right)^{2}+\left(\frac{1}{p} \int_{0}^{t_{1}} \ddot{x} \sin p t d t\right)^{2}\right)^{1 / 2}
$$

if the terms under the radical sign are integrated, the envelope of the maximum oscillator displacement at any time $t_{1}$ is given by the square root of the sum of

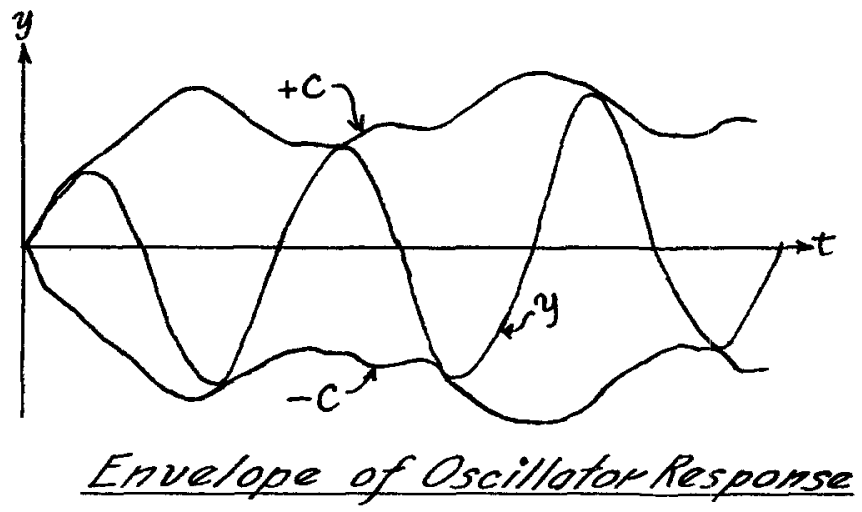

Fig. 3

the squares. When performing this integration, it is necessary to enlarge the accelerograph record and draw it to a convenient scale. The ordinates of this curve are multiplied by $\frac{\cos p t}{p}$ and the curve $\frac{\ddot{x}}{p} \cos p t$ is drawn. By means of an integraph this curve is integrated to give

$$
\int_{0}^{t_{1}} \frac{\ddot{x}}{p} \cos p t d t
$$

Repeating this procedure, using sine instead of cosine, the curre

$$
\int_{0}^{t_{1}} \frac{\ddot{x}}{p} \sin p t d t
$$

is drawn. The square root of the sum of the squares of the ordinates of these two curves gives the envelope of the maximum oscillator displacements.

The following method of arithmetical integration has also been used satisfactorily. The value of the integral

$$
\frac{1}{p} \int_{0}^{t_{1}} \ddot{x} \sin p t d t
$$


is approximated by the finite sum

$$
\frac{1}{p} \sum_{i} \ddot{x}_{\imath} \sin p t \Delta t
$$

Dividing the sin $p t$ curve into intervals of length $\Delta t$, multiplying by the corresponding ordinate $\ddot{x}_{i}$ and summing from 0 to $t_{1}$ gives the value of the integral

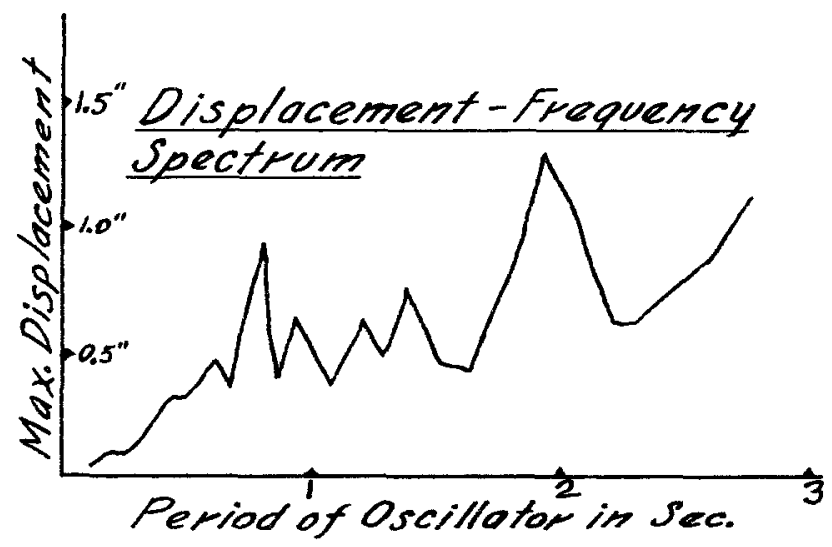

Fig. 4

to a degree of accuracy depending only upon the magnitudes of $\Delta t$. If the sin $p t$ curve is divided into increments of equal area $\Delta A$, then the summation is

$$
\frac{1}{p} \sum \ddot{x}_{i} \Delta A=\frac{\Delta A}{p} \sum \ddot{x}_{i}
$$

Making a template of the points at which the $\ddot{x}_{i}$ ordinates should be read, that is, the points of center of area of the $\Delta A$ 's, enables the $\ddot{x}_{i}$ values to be readily determined, and their sum multiplied by $\frac{\Delta A}{p}$ gives the value of

$$
\frac{1}{p} \sum \ddot{x}_{i} \sin p t d t
$$

By varying the magnitude of the factor $p$ the response of oscillators of different frequencies is determined. If the maximum displacement for each frequency is plotted as in figure 4 , the resulting curve might be called the "displacement frequency" spectrum for the earthquake.

This graph, then, indicates the sensitivity of the maximum displacements of the different frequencies to the earthquake. Large displacements may be caused either by the intensity of the earthquake, that is, the maximum accelerations, or by resonance. The "displacement frequency" spectrum gives the 
maximum displacement irrespective of whether this maxımum is a transient or a steady-state vibration so that it is perhaps more significant as an index of the destructivity of an earthquake than either the maximum acceleration occurring in an earthquake or the frequency of a predominant ground wave. The maximum displacement multiplied by $p^{2}$ gives the equivalent static force that

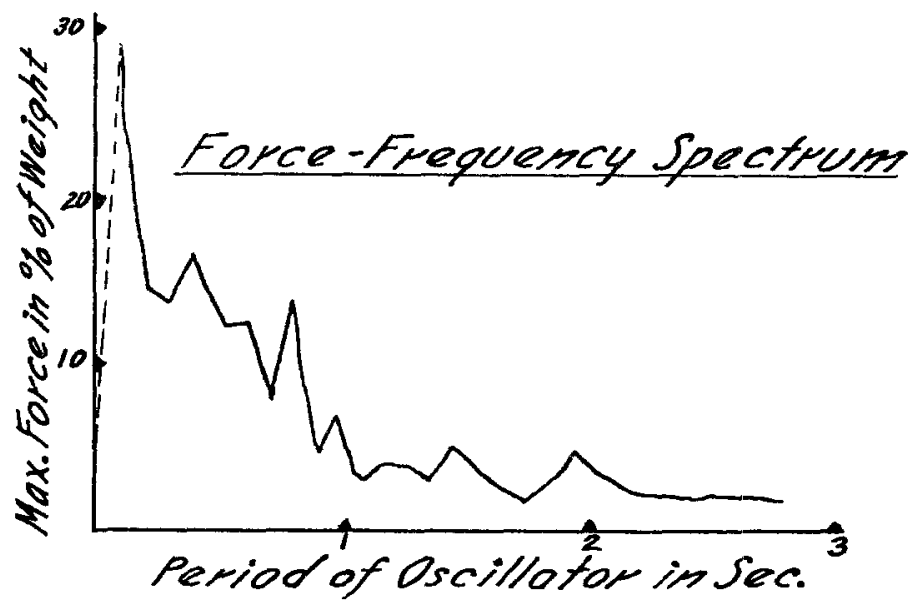

Fig. 5

would produce this displacement on an oscillator of unit mass. Plotting this equivalent force gives a "force frequency" spectrum (fig. 5). The graphs shown in figure 4 and figure 5 were computed for the earthquake of October 2, 1933, from the $\mathbf{S} 39^{\circ} \mathrm{W}$ acceleration record of the Los Angeles Subway Terminal Building seismograms.

The methods described above have been used in work done at the California Institute of Technology by Professor R. R. Martel, M. A. Biot, and M. P. White. F. Neumann of the U. S. Coast and Geodetic Survey has also used similar methods.

California Institute of Technology, Pasadena, California 